\title{
Cardiorespiratory fitness level correlates inversely with excess post-exercise oxygen consumption after aerobic-type interval training
}

Tomoaki Matsuo ${ }^{1 *}$, Kazunori Ohkawara ${ }^{2,3}$, Satoshi Seino ${ }^{4}$, Nobutake Shimojo ${ }^{4}$, Shin Yamada ${ }^{1}$, Hiroshi Ohshima ${ }^{1}$, Kiyoji Tanaka ${ }^{4}$ and Chiaki Mukai ${ }^{1}$

\begin{abstract}
Background: The purpose of this study was to reveal any association between cardiorespiratory fitness level and excess post-exercise oxygen consumption (EPOC) using three cycling protocols with varying degrees of exercise intensity, i.e., sprint interval training (SIT), high-intensity interval aerobic training (HIAT), and continuous aerobic training (CAT).
\end{abstract}

Findings: Ten healthy men, aged 20 to 31 years, attended a cross-over experiment and completed three exercise sessions: SIT consisting of 7 sets of 30-s cycling at 120\% VO $\mathrm{VO}_{2 \max }$ with a 15-s rest between sets; HIAT consisting of 3 sets of 3-min cycling at 80 90\% $\mathrm{VO}_{2 \max }$ with a 2-min active rest at $50 \% \mathrm{VO}_{2 \max }$ between sets; and CAT consisting of 40 min of cycling at $60 \sim 65 \% \mathrm{VO}_{2 \text { max }}$. During each session, resting $\mathrm{VO}_{2}$, exercise $\mathrm{VO}_{2}$, and a 180-min post-exercise $\mathrm{VO}_{2}$ were measured. The net exercise $\mathrm{VO}_{2}$ during the SIT, HIAT, and CAT averaged $14.7 \pm 1.5,31.8 \pm 4.1$, and $71.1 \pm$ $10.0 \mathrm{~L}$, and the EPOCs averaged $6.8 \pm 4.0,4.5 \pm 3.3$, and $2.9 \pm 2.8 \mathrm{~L}$, respectively. The EPOC with SIT was greater than with CAT $(P<0.01)$ and HIAT $(P=0.12)$. Correlation coefficients obtained between subjects' $\mathrm{VO}_{2 \max }$ and the ratio of EPOC to net exercise $\mathrm{VO}_{2}$ for SIT, HIAT, and CAT were $-0.61(P=0.06),-0.79(P<0.01)$, and $-0.42(P=0.23)$, respectively.

Conclusions: Our data suggest that cardiorespiratory fitness level correlates negatively with the magnitude of EPOC, especially when performing aerobic-type interval training.

Keywords: Aerobic fitness, Maximal oxygen consumption, Cycling, Energy expenditure, Exercise physiology

\section{Findings}

\section{Introduction}

A review paper regarding excess post-exercise oxygen consumption (EPOC) found that trained and conditioned individuals had a more rapid return from postexercise metabolism to resting levels after exercise [1], whereas, another review paper found that training level had minimal influence on EPOC [2]. Thus, the association between fitness level and EPOC is still controversial, and both review papers [1,2] indicate further study should be conducted to confirm the association between fitness level and EPOC for more strenuous exercise

\footnotetext{
* Correspondence: matsuo.tomoaki@jaxa.jp

'Space Biomedical Research Office, Japan Aerospace Exploration Agency (JAXA), 2-1-1, Sengen, Tsukuba, Ibaraki 305-8505, Japan

Full list of author information is available at the end of the article
}

bouts, such as levels greater than $80 \%$ maximal oxygen consumption $\left(\mathrm{VO}_{2 \max }\right)$.

We have been developing two types of original exercise training protocols at the Japan Aerospace Exploration Agency (JAXA) which are better suited to astronauts during long-term spaceflight: sprint interval training (SIT) and high-intensity interval aerobic training (HIAT). In a previous study [3], we compared total energy expenditure (EE), including EPOC, induced by the two protocols with the total EE of traditional, continuous aerobic training (CAT). The study showed that exercise intensity had a noticeable effect on the EPOC. That is, although the SIT was the shortest duration among our three protocols, and the net exercise EE of the SIT (77 kcal) was only $20 \%$ of the EE of CAT $(350 \mathrm{kcal}$ ) and $50 \%$ of the EE of HIAT (161 kcal), SIT's EPOC during the 180-min

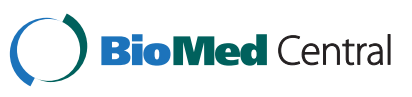


post-exercise period was the greatest among the three protocols. In this short report, we examine associations between a subject's cardiorespiratory fitness level and EPOC using our three exercise protocols.

\section{Methods}

Detailed descriptions of the study protocol have been published elsewhere [3]. Briefly, our ten male subjects' average values in age, height, body weight, body mass index and $\mathrm{VO}_{2 \max }$ were $24.0 \pm 3.3$ (range: $20-31$ ) years, $170.8 \pm 5.0(162.1-175.6) \mathrm{cm}, 61.9 \pm 5.7(54.6-69.7) \mathrm{kg}$, $21.2 \pm 1.7(18.4-23.3) \mathrm{kg} \cdot \mathrm{m}^{-2}$, and $3.18 \pm 0.45(2.34-3.75)$ $\mathrm{L} \cdot \mathrm{min}^{-1}$ (or $52.0 \pm 9.2\left(36.2\right.$ - 61.4) $\mathrm{ml} \cdot \mathrm{kg}^{-1} \cdot \mathrm{min}^{-1}$ ), respectively. The aim and design of this study were explained to every subject before each gave their written, informed consent. This study was conducted in accordance with the guidelines proposed in the Declaration of Helsinki. The Ethical Committee of both JAXA (reference number: 32-27) and University of Tsukuba (22-283) reviewed and approved the study protocol.

The subjects completed three experimental sessions separated by approximately 1 week. During each session, the subjects performed one of three exercise trials, i.e., SIT, HIAT, or CAT. The detailed protocols of the three cycling exercises are shown in Additional file 1: Table S1. All aspects of the experimental session remained the same during all three sessions except the actual exercise technique. The three sessions were implemented in random order.

On each session day, the subjects arrived at our laboratory at approximately 7:15 am. They were asked to minimize any physical activity (walking etc.) while en route from their home to the laboratory. Six subjects drove their own cars to the laboratory, and 4 subjects were picked up at their homes and driven to the building by a research assistant because they did not have their own cars. At 7:45 am, a subject would seat himself in a comfortable armchair and remain in a resting (seated) position without movement until 8:20 am. From 8:05 to 8:20 am (15 minutes), the subject was connected to a face mask of the indirect calorimeter for baseline data collection. Average $\mathrm{VO}_{2}$ data during the final 10 minutes were used as the baseline value. After the baseline measurements, we disconnected the face mask and allowed the subject to take a drink of water before riding on the cycling ergometer (75XL Ш, Konami, Tokyo, Japan) for the exercise phase. After the water, he was reconnected to the indirect calorimeter and started pedaling the ergometer at 8:25 am.

After the exercise phase, the subject returned to the resting position immediately but stayed connected to the indirect calorimeter for 10 minutes to measure immediate post-exercise $\mathrm{VO}_{2}$. After 10 minutes, we removed the face mask but the subject remained in the resting position for another 170 minutes. During the postexercise phase, the subject remained in the resting position immobile. Ten minutes of $\mathrm{VO}_{2}$ measurements were started at $30,60,90,120,150$, and 180 minutes post exercise. For each measurement session, average $\mathrm{VO}_{2}$ data during the final 5 minutes was used.

All gas exchange measurements were measured using the mixing chamber method with an open-circuit computerized indirect calorimeter (AE-310S, Minato Medical Science, Osaka, Japan). The ventilatory volume, $\mathrm{VO}_{2}$, and $\mathrm{VCO}_{2}$ were calculated every 15 seconds. The gas analyzer was calibrated before each trial. Coefficient of variation $(\mathrm{CV})$ from the mean for the three resting $\mathrm{VO}_{2}$ measurements in the 10 subjects was $4.3 \%$.

To measure each subject's $\mathrm{VO}_{2 \max }$, we used the criteria described by Tanaka et al. [4] Briefly, after a 2-min warm up at 15 Watt (W), the subjects began the actual exercise protocol at a $30 \mathrm{~W}$ level. The workload was increased every minute by $15 \mathrm{~W}$ until volitional exhaustion. We determined the highest oxygen uptake achieved over 30 seconds as the $\mathrm{VO}_{2 \max }$.

To determine each subject's exercise intensity during the exercise session, we used the $\mathrm{VO}_{2 \max }$ measurement data (i.e., values per minute for workload and $\mathrm{VO}_{2}$ ) and calculated a simple linear regression equation for each subject: $\mathrm{Y}=\beta \mathrm{x}+\mathrm{c}$, with $\mathrm{Y}=$ workload $(\mathrm{W}), \mathrm{x}=\mathrm{VO}_{2}(\mathrm{ml})$, and $\beta$ and $c$ as constants. Subsequently, percentage $\mathrm{VO}_{2 \max }$ data (ml) (e.g., $120 \% \mathrm{VO}_{2 \max }$ for SIT) were applied to the equation, whereupon each subject's exercise intensity (workload (W)) was determined.

To determine net exercise $\mathrm{VO}_{2}$ and EPOC, the area under the curve (AUC) for each phase was calculated for each subject using a statistical-software package (SAS version 9.2, SAS Institute Inc, Cary, NC, USA). As for the SIT and HIAT, periods of active rest were included in the calculation of the net exercise $\mathrm{VO}_{2}$.

To analyze differences among the three sessions, we used one-way analysis of variance (ANOVA), and applied Tukey-Kramer's post hoc test when the difference was significant $(P<0.05)$ according to the results of ANOVA. We assessed the relationship between two measurement values with Pearson's product moment correlation. We used SAS, version 9.2 (SAS Institute Inc, Cary, NC, USA) to analyze the data.

\section{Results}

The net $\mathrm{VO}_{2}$ incurred with the HIAT $(31.8 \pm 4.1 \mathrm{~L})$ was significantly lower than during the CAT $(71.1 \pm 10.0 \mathrm{~L})$, but it was significantly greater than the net $\mathrm{VO}_{2}$ incurred during the SIT $(14.7 \pm 1.5 \mathrm{~L})$. The EPOC was significantly greater with the SIT $(6.8 \pm 4.0 \mathrm{~L})$ than with the CAT $(2.9 \pm 2.8 \mathrm{~L})$, and it also tended to be greater than with the HIAT $(4.5 \pm 3.3 \mathrm{~L})(P=0.12)$. We 
observed no significant differences in the EPOC between HIAT and CAT.

Additional file 2: Figure S1 shows the relationship between subjects' $\mathrm{VO}_{2 \max }$ and the ratios of EPOC to the net exercise $\mathrm{VO}_{2}$ during each exercise session. The ratio of EPOC to net exercise $\mathrm{VO}_{2}$ during the SIT $(r=-0.61$, $P=0.06)$ and the HIAT $(r=-0.79, P<0.01)$ correlated inversely with $\mathrm{VO}_{2 \max }$, whereas, we observed no significant correlation between these two variables for the CAT $(r=-0.42, P=0.23)$. As for the correlation between absolute EPOC value and $\mathrm{VO}_{2 \max }$, we observed a significant correlation for the HIAT $(r=-0.67, P=0.03)$, but no significant correlation for the SIT $(r=-0.47$, $P=0.17)$ and CAT $(r=-0.34, P=0.34)$.

\section{Discussion}

Ohkawara et al. [5] showed a significant negative correlation between a subject's fitness level and the EPOC under normal living conditions in a metabolic chamber. The significant correlation was observed not on a day with moderate-intensity physical activity but on a day with vigorous-intensity physical activity. Also, Singh et al. [6] recently showed that $\mathrm{VO}_{2}$ decline during the first minute of recovery after maximum exercise was faster in children with a higher $\mathrm{VO}_{2 \max }$. Our experimental results with a single bout of exercise were consistent with those studies' results. That is, in the present study, while the ratio of EPOC to net exercise $\mathrm{VO}_{2}$ correlated inversely with $\mathrm{VO}_{2 \max }$, we observed this significant association when subjects performed a high-intensity interval exercise, especially an aerobic-type interval protocol (the HIAT), but not when they performed a moderateintensity continuous protocol (the CAT). Although several studies [7-9] reported that training level did not significantly impact EPOC, the exercise intensities (30 70\% $\left.\mathrm{VO}_{2 \max }\right)$ used in those previous studies were relatively low compared to our interval exercise protocols.

While the precise mechanism of EPOC remains unclear, physiological adaptation associated with improved fitness levels has been considered a factor potentially influencing EPOC [1]. For example, an enhanced lactate metabolism with elevated body temperature partly accounts for EPOC $[2,10]$. Trained individuals have better thermoregulatory capacities than untrained individuals because physical training enhances the sweating mechanism at a given level of the central sweating drive [11]. Therefore, elevated body temperature in untrained individuals could last longer than in trained individuals [12]. Moreover, subjects with lower $\mathrm{VO}_{2 \max }$ might produce more lactate than those with higher $\mathrm{VO}_{2 \max }$ especially during strenuous exercise. An enhanced lactate metabolism requires oxygen consumption for recovery. Thus, fitness level may contribute to the magnitude of EPOC.
In conclusion, we revealed that cardiorespiratory fitness level correlates inversely with the magnitude of EPOC, especially when performing an aerobic-type interval exercise.

\section{Additional files}

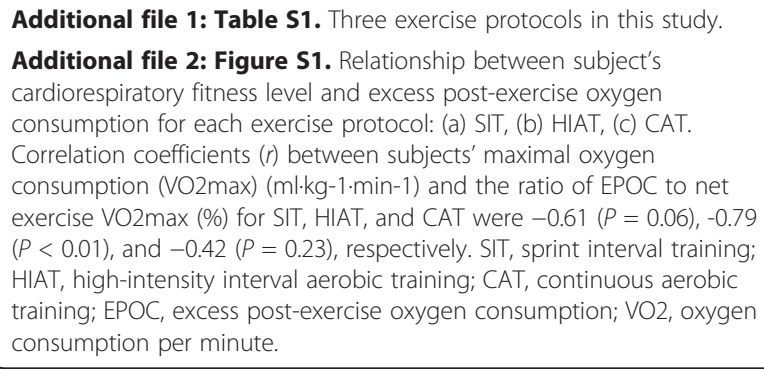

\section{Competing interest}

The authors declare no conflict of interest.

\section{Authors' contributions}

The contributions of each author were as follows: TM and $\mathrm{KO}$ contributed to manuscript writing, developing study concept and design, data acquisition, and data analysis; SS contributed to manuscript revisions, data acquisition, and data analysis; NS contributed to manuscript revisions and data acquisition; SY and $\mathrm{HO}$ contributed to manuscript revisions and developing study concept and design; KT and CM represented the University of Tsukuba and JAXA, respectively, in this joint research project and contributed to manuscript revisions, developing study concept and design, and data acquisition. All authors read and approved the final manuscript.

\section{Acknowledgments}

We are grateful to the participants in this study. We thank Drs. Toshiko Ohta, Ken-ichi Iwasaki, Masamichi Sudoh, and Messrs. Naoya Oki, Tatsuya Aiba, Hajime Takeoka, and Masafumi Yamamoto for their support with this study. The study was jointly accomplished through JAXA and the University of Tsukuba and supported by JAXA funding for 2010 Space Biomedical Research projects.

\section{Author details}

${ }^{1}$ Space Biomedical Research Office, Japan Aerospace Exploration Agency (JAXA), 2-1-1, Sengen, Tsukuba, Ibaraki 305-8505, Japan. ${ }^{2}$ Faculty of Informatics and Engineering, University of Electro-Communications, 1-5-1 Chofugaoka, Chofu, Tokyo 182-8585, Japan. ${ }^{3}$ Health Promotion and Exercise Program, National Institute of Health and Nutrition, 1-23-1, Toyama, Shinjuku, Tokyo 162-8636, Japan. ${ }^{4}$ Graduate School of Comprehensive Human Sciences, University of Tsukuba, 1-1-1 Tennodai, Tsukuba, Ibaraki 305-8574, Japan.

Received: 9 July 2012 Accepted: 18 October 2012 Published: 21 November 2012

\section{References}

1. Borsheim E, Bahr R: Effect of exercise intensity, duration and mode on post-exercise oxygen consumption. Sports Med 2003, 33:1037-1060.

2. LaForgia J, Withers RT, Gore CJ: Effects of exercise intensity and duration on the excess post-exercise oxygen consumption. J Sports Sci 2006, 24:1247-1264.

3. Matsuo T, Ohkawara K, Seino S, Shimojo N, Yamada S, Ohshima H, Tanaka K, Mukai C: An exercise protocol designed to control energy expenditure for long-term space missions. Aviat Space Environ Med, in press.

4. Tanaka K, Takeshima N, Kato T, Niihata S, Ueda K: Critical determinants of endurance performance in middle-aged and elderly endurance runners with heterogeneous training habits. Eur J Appl Physiol Occup Physiol 1990, 59:443-449. 
5. Ohkawara K, Tanaka S, Ishikawa-Takata K, Tabata I: Twenty-four-hour analysis of elevated energy expenditure after physical activity in a metabolic chamber: models of daily total energy expenditure. Am J Clin Nutr 2008, 87:1268-1276.

6. Singh TP, Alexander ME, Gauvreau K, Curran T, Rhodes Y, Rhodes J: Recovery of oxygen consumption after maximal exercise in children. Med Sci Sports Exerc 2011, 43:555-559.

7. Freedman-Akabas S, Colt E, Kissileff HR, Pi-Sunyer FX: Lack of sustained increase in VO2 following exercise in fit and unfit subjects. Am J Clin Nutr 1985, 41:545-549.

8. Brehm BA, Gutin B: Recovery energy expenditure for steady state exercise in runners and nonexercisers. Med Sci Sports Exerc 1986, 18:205-210.

9. Sedlock DA: Fitness level and postexercise energy expenditure. J Sports Med Phys Fitness 1994, 34:336-342.

10. Bahr R, Gronnerod O, Sejersted OM: Effect of supramaximal exercise on excess postexercise O2 consumption. Med Sci Sports Exerc 1992, 24:66-71.

11. Astrand P, Rodahl K: Textbook of work physiology: physiological bases of exercise. 2nd edition. New York: McGraw-Hill Book Company; 1977.

12. Frey GC, Byrnes WC, Mazzeo RS: Factors influencing excess postexercise oxygen consumption in trained and untrained women. Metabolism 1993, 42:822-828.

doi:10.1186/1756-0500-5-646

Cite this article as: Matsuo et al:: Cardiorespiratory fitness level

correlates inversely with excess post-exercise oxygen consumption after aerobic-type interval training. BMC Research Notes 2012 5:646.

\section{Submit your next manuscript to BioMed Central and take full advantage of:}

- Convenient online submission

- Thorough peer review

- No space constraints or color figure charges

- Immediate publication on acceptance

- Inclusion in PubMed, CAS, Scopus and Google Scholar

- Research which is freely available for redistribution 